\title{
Temporal Modeling of Node Mobility in Mobile Ad-hoc Network
}

\author{
Jyoti Prakash Singh ${ }^{1}$ and Paramartha Dutta ${ }^{2}$ \\ ${ }^{1}$ Department of Information Technology, Academy of Technology, West Bengal,India \\ ${ }^{2}$ Department of Computer and System Sciences, Visva-Bharati University, West Bengal, India
}

\begin{abstract}
Ad-hoc network consists of a set of identical nodes that move freely and independently and communicate via wireless links. The most interesting feature of this network is that it does not require any predefined infrastructure or central administration and hence it is very suitable for establishing temporary communication links in emergency situations. This flexibility however is achieved at the price of communication link uncertainties due to frequent topology changes. In this article we describe the system dynamics using the proven concept of time series modeling. Specifically, we analyze variations of the number of neighbor nodes of a particular node over a geographical area and for given total number of nodes assuming different values of (i) the speeds of nodes, (ii) the transmission powers, (iii) sampling periods and (iv) different mobility patterns. We consider three different mobility models: (i) Gaussian mobility model, (ii) random walk mobility model and (iii) random way point mobility model. The number of neighbor nodes of a particular node behaves as a random variable for any mobility pattern. Through our analysis we find that the variation of the number of neighbor nodes can be well modeled by an autoregressive $\operatorname{AR}(p)$ model. The values of $p$ evaluated for different scenarios are found to be in the range between 1 and 5. Moreover, we also investigate the relationship between the speed and the time of measurements, and the transmission range of a specific node under various mobility patterns.
\end{abstract}

Keywords: ad-hoc network, mobility modeling, time series analysis, autoregressive modeling

\section{Introduction}

Multi-hop wireless networks [10, 14], commonly referred to as the ad-hoc wireless networks do not require fixed infrastructure since the mobile nodes can relay their packets to other nodes without using base stations. The nodes are mobile and change their locations regularly. A node in mobile ad-hoc network (MANET) is moving and its neighbors are changing constantly with time and location. The number of neighbor nodes of a node is important information for several network services such as network connectivity, routing, congestion control, topology construction, security etc as discussed in [20]. The number of neighbor nodes is also important information for the MAC protocol design. There will be increased contention among the neighboring node with increasing number of neighbors of a particular node. This may be used for the purpose of predicting the future neighborhood structure of the node, for example, to optimize the back-off period of the MAC protocol for this node. Let $\mathrm{N}=\left\{N_{i} \mid 1 \leq i \leq n\right\}$ represents the nodes in the network. The neighbor count $(\mathrm{NC})$ of the node $\mathrm{N}_{i}$ is a random variable. At every time instant, some new nodes are entering the transmission range of $\mathrm{N}_{i}$ while some existing neighbor nodes are leaving the transmission range of $\mathrm{N}_{i}$. Say, the node $\mathrm{N}_{i}$ has $\mathrm{NC}_{i}$ many number of nodes at time instant $t$. After a small duration of time $\Delta t$, i.e., at time $\mathrm{t}+\Delta \mathrm{t}$, let this number of nodes be $\mathrm{NC}_{t+\Delta t}$. $\mathrm{NC}_{t+\Delta t}$ is a function of the previous neighbor counts $\mathrm{NC}_{t}, \mathrm{NC}_{t-\Delta t}, \ldots$ due to the fact that some existing nodes have left while new nodes have come into the transmission range. How many nodes leave and come is a random phenomenon [21] which is dependent on (i) the speed of the node $\mathrm{N}_{i}$ and its neighbors, (ii) the transmission range of the node $\mathrm{N}_{i}$ and its neighbors, (iii) the time interval when we take the observations and (iv) the mobility pattern $[9,25]$ followed by the node $\mathrm{N}_{i}$ and its neighbors. If the nodes move very slowly or they are almost stationary, then the correlation between the ex- 
isting and new value of the neighbor count $\mathrm{NC}$ will be very high. Correspondingly, we define $\mathrm{NC}_{t+\Delta t}$ as a function of $\mathrm{NC}_{t}, \mathrm{NC}_{t-\Delta t}, \mathrm{NC}_{t-2 \Delta t}$, $\ldots \mathrm{NC}_{t+\Delta t}=f\left(\mathrm{NC}_{t}, \mathrm{NC}_{t-\Delta t}, \ldots, \mathrm{NC}_{t-p \Delta t}\right)$ for some integer $p$ such that $1 \leq p<\infty$. The autocorrelation of NC also depends on speed, sampling time, transmission range and mobility patterns. If the speed is low, the nodes move a short distance within a given duration. Most of the nodes which were neighbors in earlier time instances are expected to remain neighbors again, assuming the fixed transmission range. Very few nodes move out of the transmission range and very few new nodes arrive in. If the speed is increased, the nodes move longer distances and may come out of the transmission range. Similarly, several other new nodes may come within the transmission range of the node $\mathrm{N}_{i}$ and become its neighbors. Since large number of nodes are leaving and arriving with very few existing neighbor nodes remaining, the correlation between the existing neighbor count value $\mathrm{NC}_{\text {old }}$ and the new neighbor count value $\mathrm{NC}_{\text {new }}$ does not remain as high as it used to be in the case of nodes that move slowly.

The transmission range defines the area where the packets sent by the node can be received correctly by other nodes with a high probability. This area is usually represented by a circle about each node. The radius of this circle is proportional to the square root of the transmitting power. If the transmission power of a node is small, i.e., the transmission range is also small, then the neighbor count will vary more abruptly. This is because if the node moves to a region of high nodes density, then momentarily its number of neighbors will be very high, however, a small change in location in the next time instant may leave this node with very few or even no neighbors at all. In other words, the autocorrelation between $\mathrm{NC}$ values is very low if the transmission power (and hence the transmission range) are also low. On the other hand, the autocorrelation between $\mathrm{NC}$ values will increase with increasing the transmission power. The time separation of periodical neighbor counting is termed as sampling time. The variation in neighbor count value $\mathrm{NC}$ is also a function of the nodes locations. The distance the node moves depends on two factors: (i) the node speed and (ii) the sampling time. Even for moderate speeds, if the sampling time is increased, several nodes move out of the node range while several other nodes arrive. Due to large number of nodes movements, the time correlations of the NC values do not remain very strong. Thus the autocorrelation of NC decreases as the sampling time is increased. In this article, we consider three mobility models: (i) Gaussian, (ii) random walk and (iii) random way point $[25,1,11,5,17,16,6]$. A survey of the most frequently used mobility models can be found in [9]. More importantly, we find that the effect of particular mobility pattern is insignificant with respect to the autocorrelation of the $\mathrm{NC}$ values for a given node and different time periods. The random walk and random way point mobility models are found to exhibit stronger autocorrelations between $\mathrm{NC}$ values at higher speeds than the Gaussian mobility model; however, even then the difference is quite small and may be neglected in most cases.

The main aim of this article is to investigate statistical behavior of the number of neighbor nodes in a mobile ad-hoc network. The number of neighbors can be utilized to other areas such as augmenting the performance of existing routing protocols, enhancing security issues etc. The rest of this article is organized as follows. Section 2 contains a brief survey of the relevant literature. Section 3 introduces the autoregressive (AR) model of order $p$. In Section 4, we present the simulation results to justify the proposed $\operatorname{AR}(p)$ model. Section 5 describes the techniques to find the order $p$ of the AR model. The predicted values using the developed AR model are also given. In Section 6, we conclude and provide suggestions for future research.

\section{Related Works}

Time series $[12,8]$ has attracted a lot of attention in modeling of internet traffic, wireless sensor and ad-hoc network traffic. Basu et al. [4] models the internet traffic using the autoregressive moving average (ARMA) of order $p$ and $q$. Using this model, they predict the traffic which is generated by a TCP source using FDDI protocol. They also develop a generator of synthetic traffic that is useful for simulation studies of internet traffic and in resource management algorithms. You and Chandra [24] use statistical means to show that the aggregate TCP packet arrival process exhibits non-stationary and nonlinear features. They generate stationary traffic 
by filtering a subset of the processes exhibiting non-stationary features from the aggregate process. Such a filtered traffic process is modeled using non-linear threshold autoregressive processes. This traffic model is found to be in a good agreement with the real traffic assuming the packet loss statistics. This model can be used in the design of traffic shapers providing a simple and accurate approach for simulating internet data traffic patterns. Liu et al. [19] propose an energy efficient technique for information collection in sensor nodes. They hold back the sensor nodes from transmitting redundant data. The data is redundant if it can be predicted by the sink node. For prediction, they utilize AutoRegressive Integrated Moving Average (ARIMA) model [8] of order (p, d, q) due to its outstanding model fit and small computational cost. The samples from a specific sensor node arriving at the sink node are treated as time series and the sink maintains the time series for each sensor node. The time series are then used for predictions by sink nodes for each of the sensor nodes. If the difference between the actual data and the predicted data is within a pre-defined threshold, then this data are not sent from the sensor node, thus providing energy savings. Herbert et al. [15] extend the idea of Liu to cluster-based sensor network. The LEACH [13] protocol is extended to provide verification at the clusterhead. Each member node transmits the AutoRegressive Integrated Moving Average (ARIMA) parameters to its clusterhead which verifies the accuracy of the model by generating time series with each set of parameters and then calculate the mean squared error of the predicted data. If the mean squared error is above a fixed tolerance value, the clusterhead requests all member nodes to recalculate their respective parameters repeatedly until all the models are within a tolerance. Borgne et al. [7] use a set of time series models to predict the sensors reading at regular intervals of time by the sink node. The sink node transmits this data to every sensor. If sensor nodes find that their readings are different from the sink node's predictions by a value greater than a given threshold, then the sensors send their readings to the sink. This approach has shown great savings in the communication cost of the sensor networks. Banerjee et al. [2,3] model the system dynamics using birth and death model. The node entering in the transmission range of a source node is treated as birth of this particular node. Similarly, when the node leaves the transmission range of the sink node, it is considered to be the death of this node. Finally, [18] presents a typical ad-hoc network topology and derives the statistical properties of neighbor nodes for that topology.

\section{Proposed Model}

As pointed out in Section 1, the neighbor count $\mathrm{NC}$ of a node $\mathrm{N}_{i}$ is randomly changing over time and is a function of $\mathrm{NC}$ values at previous time instances. In this article, we describe the network mobility dynamics using the concepts of time series. The time series $[12,8]$ are sequences of observations ordered according to the time of their occurrences. By recording and analyzing the data of time series, we can gain a better understanding of the underlying data generating mechanism and also make predictions about the future data. The main characteristic of time series is that the data are often governed by a trend having cyclic components. An important step for analyzing the time series $[12,8]$ is the proper selection of a suitable model (or class of models) fitting the observed data; i.e., the more appropriate is the model selection, the better are the predictions. The neighbor count $\mathrm{NC}$ of node $\mathrm{N}_{i}$ and the their correlations in time are important parameters to describe the models of nodes movements. For instance, the plots of experimental measurements of the values of $\mathrm{NC}$ confirm that the autocorrelations are very high at initial lags and are monotonically decreasing with larger lags. This implies that the current neighbor count $\mathrm{NC}$ is a function of the values of $\mathrm{NC}$ at previous time instances. Hence we propose to model the nodes movements by an $\operatorname{AR}(p)$ model $[12,8]$. The $\operatorname{AR}(p)$ model is defined as,

$$
r_{t}=\phi_{0}+\phi_{1} r_{t-1}+\phi_{2} r_{t-2}+\ldots+\phi_{p} r_{t-p}+a_{t}
$$

where $p$ is a non-negative integer, $a_{t}$ are the samples of white noise with mean zero and variance $\sigma^{2}$, and $\phi_{i}$ are the time-invariant coefficients. This model suggests that the past $p$ values $r_{t-i}$, for $i=1, \cdots, p$, are correlated. The time series considered in the experiments in this article are stationary since the following two conditions hold [12]: 
1. $E\left(r_{t}\right)=\mu$ is constant independent of time instant $t$

2. $\operatorname{Cov}\left(r_{t}, r_{t-j}\right)=\gamma^{j}$ only depends on time $\operatorname{lag} j$.

Then, the mean value of these series is given as

$$
E\left(r_{t}\right)=\frac{\phi_{0}}{1-\phi_{1}-\phi_{2}-\ldots-\phi_{p}} .
$$

The autocovariance is given as

$$
\gamma^{j}=\left\{\begin{array}{c}
\phi_{1} \gamma^{j-1}+\phi_{2} \gamma^{j-2}+\ldots+\phi_{p} \gamma^{j-p} \\
j=1,2, \ldots p \\
\phi_{1} \gamma^{1}+\phi_{2} \gamma^{2}+\ldots+\phi_{p} \gamma^{p} \\
j=0
\end{array}\right.
$$

Also, if all the roots of the characteristic function of the AR model

$$
x^{p}-\phi_{1} x^{p-1}-\phi_{2} x^{p-2}-\ldots-\phi_{p}
$$

are less than unity in modulus, then the series $r_{t}$ is stationary [12]. Depending on the nature

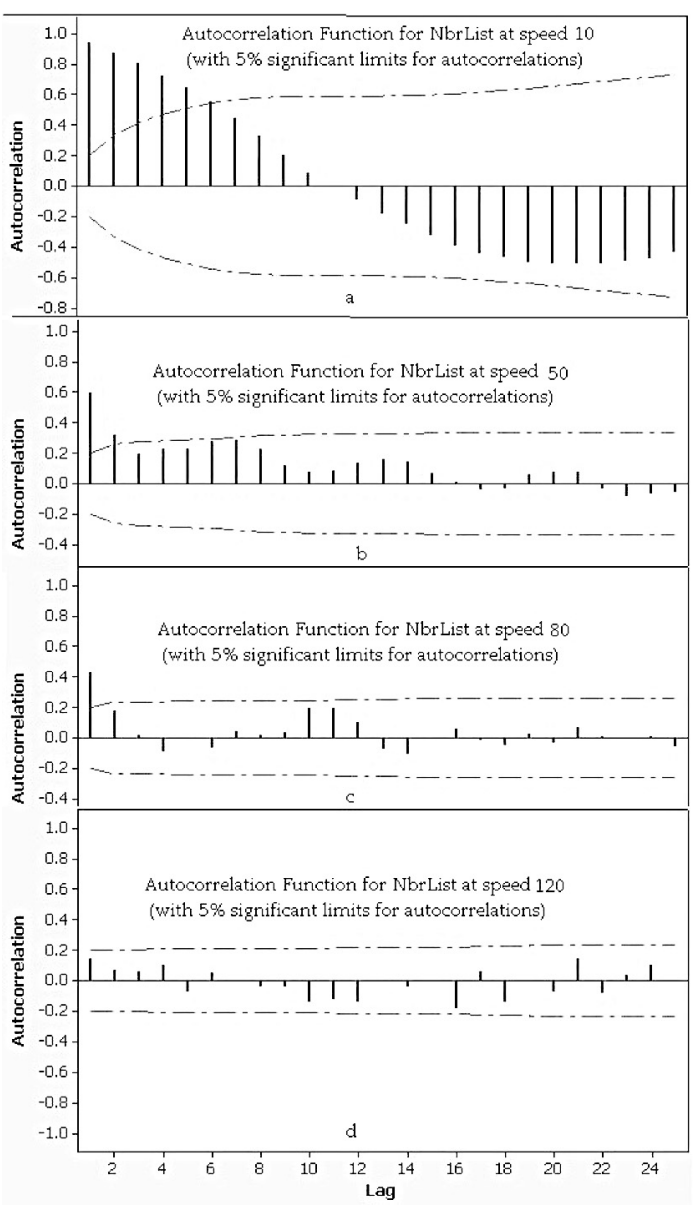

Figure 1. Variation of the NC autocorrelation with speed for the Gaussian mobility pattern. of the roots, the characteristic function can indicate if the autocorrelation function (ACF) of the $\operatorname{AR}(p)$ model contains a mixture of damping sine and cosine patterns and exponential decays. This is verified by our experimental data. We have to also choose the proper value of $p$ to represent data using the $\operatorname{AR}(p)$ model. In this article, we use the partial autocorrelation function (PACF) to find the order the of AR model. We then also use the Akeike Information Criterion (AIC) [8] to confirm the proper choice of $p$.

\subsection{Performance Consideration}

Our AR predictions are based on 100 past measurements. Thus, each node has to store 100 integer values assuming that each node is represented by an integer, so that the storage requirements are 200 bytes. The order of AR model

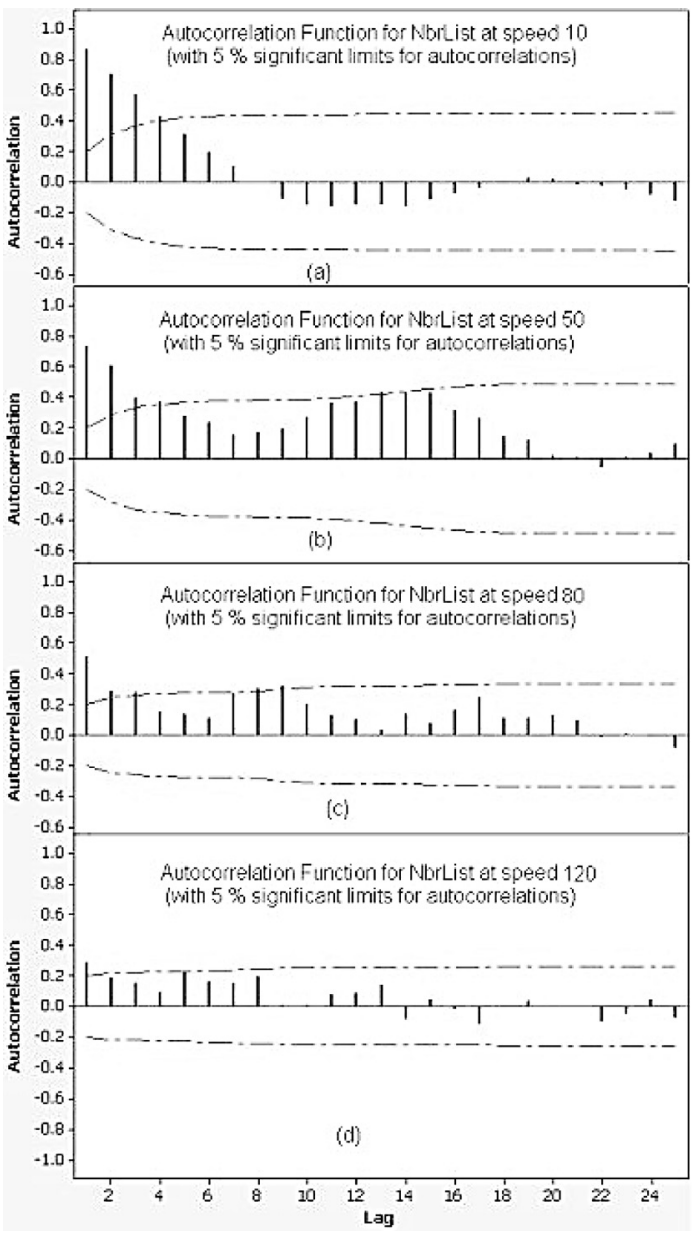

Figure 2. Variation of the NC autocorrelation with speed for the random walk mobility pattern. 
for our system is found to be between 2 and 3 for most of the cases considered. For the 3rd order AR model, we need matrices of the order $100 \times 3$ to solve for the coefficients and for subsequent predictions. Hence, the computational overhead of our model is not of concern and it can be easily used by each network node.

\section{Simulation and Results}

We have used Omnet ++ [22] for node mobility simulation while Minitab is used to analyze the results.

\subsection{Simulation Setup}

The node mobility and traffic generations are simulated using Omnet ++3.3 [22] discrete event system simulator considering 131 mobile

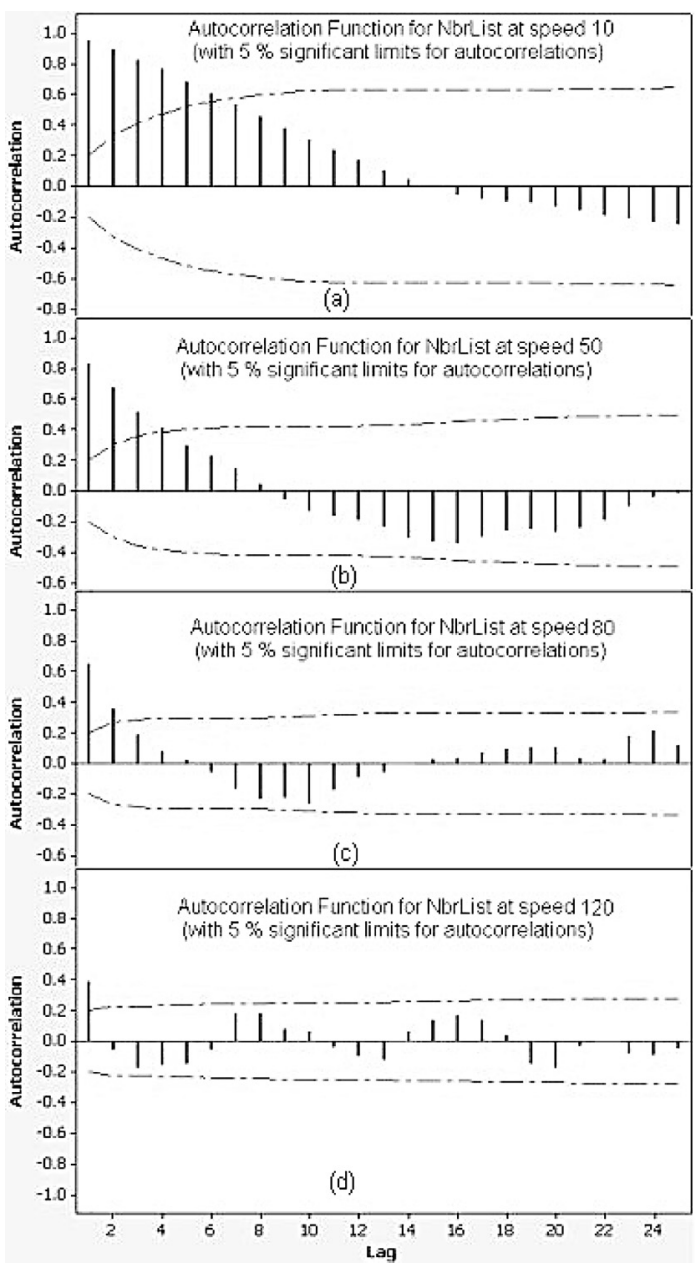

Figure 3. Variation of the NC autocorrelation with speed for the random way point mobility pattern. nodes. We have analyzed the neighbor count for three mobility models: Gaussian, random walk and random way point. The nodes are distributed over an area of $600 \times 600$ meters. The speed of the node movement varies between 10 meters per second $(\mathrm{m} / \mathrm{sec})$ to $170 \mathrm{~m} / \mathrm{sec}$. The transmission range varies from 1000 to 90000 meters; the coverage area is the square root of the transmission power. The number of neighbors of each node is studied at $0.5,1$ and 1.5 seconds for each value of the node speeds. Standard network protocol has been used for the simulations. The results are processed in Minitab version 14 to obtain the statistical analysis. Yoon and Liu prove mathematically [23] that the average speed of nodes using random way point mobility model decreases constantly and would eventually reach zero. To overcome this problem, they suggest to use a non zero minimum speed. Hence, we have always set the minimum speed $V_{\min }$ of the nodes to a positive value for all mobility models considered.

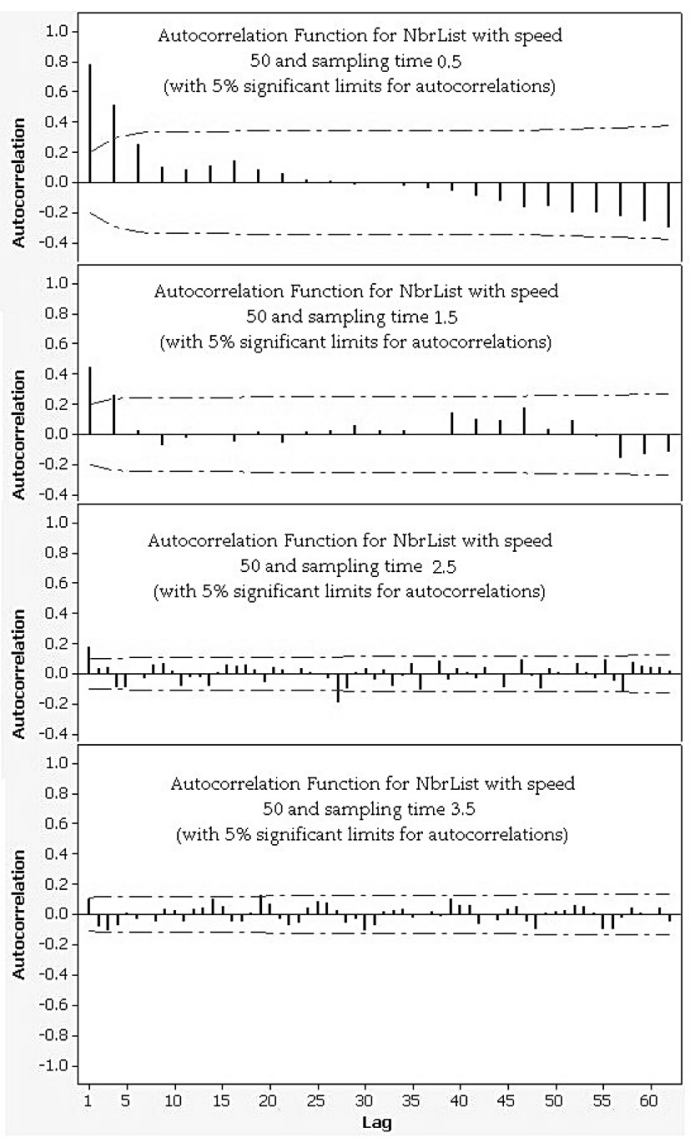

Figure 4. Variation of the NC autocorrelation with sampling time for the Gaussian mobility pattern. 


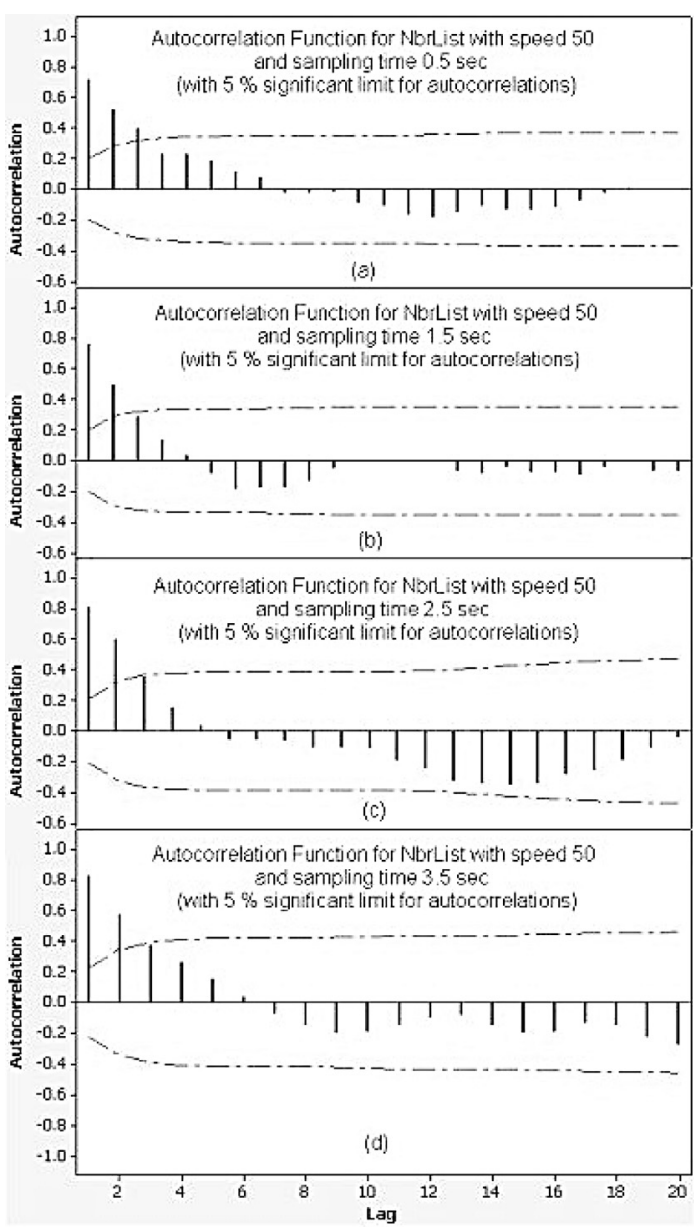

Figure 5. Variation of the NC autocorrelation with sampling time for the random way point mobility pattern.

\subsection{Analysis for Varying Speed and a Fixed Sampling Rate}

In our first experiment, we find the relationship between the autocorrelation of NC and the speed of moving nodes. We assume that all nodes are moving at equal speed and the readings are taken in regular intervals of 1 second. At low speed, the NC shows high autocorrelation between several previous lagged values. However, as the speed increases, more nodes start to leave and join the transmission regions, and so the autocorrelation between the previous values diminishes.

We computed the autocorrelations among NC data for nodes moving at the speed of $10 \mathrm{~m} / \mathrm{sec}$. The speed was varied from 10 to $120 \mathrm{~m} / \mathrm{sec}$ with increments of $10 \mathrm{~m} / \mathrm{sec}$. The samples are taken every $1 \mathrm{sec}$ in each case. Some of the samples correlations at speeds $10,50,80$ and $120 \mathrm{~m} / \mathrm{sec}$

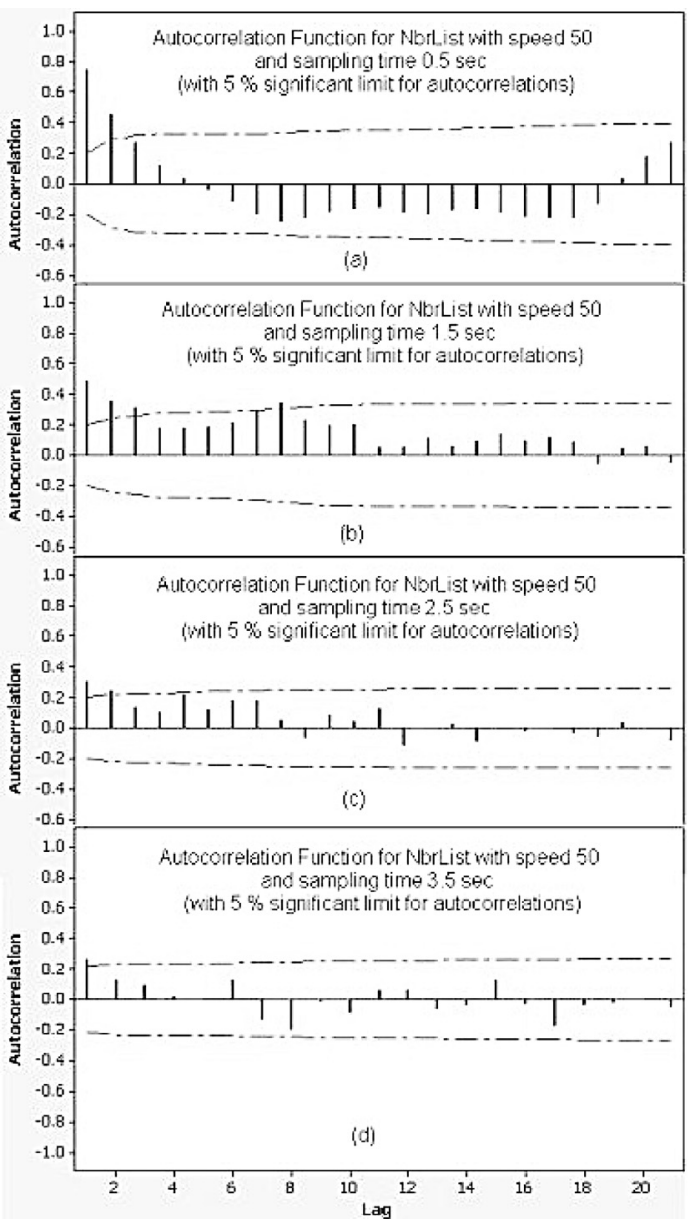

Figure 6. Variation of the NC autocorrelation with sampling time for the random walk mobility pattern.

are shown in Figure 1. As evident from Figure 1.a, the $\mathrm{NC}$ values for nodes moving at the speed of $10 \mathrm{~m} / \mathrm{sec}$ have high autocorrelations with the high peaks at initial lag values, and the autocorrelations are decreasing for higher lags. However, when the speed reaches 120 $\mathrm{m} / \mathrm{sec}$, no significant autocorrelation exists as evident from Figure 1.d. Figure 1 shows an example of the autocorrelation of the NC values for the Gaussian mobility model. For other two models the results are very similar, as shown in Figures 2 and 3. The autocorrelations become insignificant at the speed of $130 \mathrm{~m} / \mathrm{sec}$ for the random way point mobility pattern whereas for the random walk mobility pattern, it remains significant up to the speed of $200 \mathrm{~m} / \mathrm{sec}$. Thus, the random walk mobility model shows strong autocorrelation between the NC values at higher speeds in comparison to the other two mobility models considered. 


\subsection{Analysis for Constant Speed and Varying Sampling Rate}

In our second experiment, we measure the variations of the number of neighbors with sampling time. For the nodes moving very slowly, the neighborhoods are also moving very slowly. In this case, when the data are sampled frequently, we obtain nearly similar number of neighbors. For this experiment, we have sampled the data between $0.5 \mathrm{sec}$ to $5 \mathrm{sec}$ with a sampling period of $0.5 \mathrm{sec}$. We assume the fixed speed 50 $\mathrm{m} / \mathrm{sec}$. The autocorrelation ACF for nodes following the Gaussian mobility is shown in Figure 4. For sampling time of $0.5 \mathrm{sec}$, we get the ACF which is sinusoidal in nature and has higher autocorrelation values at initial lags. However, for sampling times larger than $3.5 \mathrm{sec}$, no autocorrelation values are significant, as evident from Figure 4.d. Figures 5 and 6 illustrate the autocorrelations versus sampling time for the

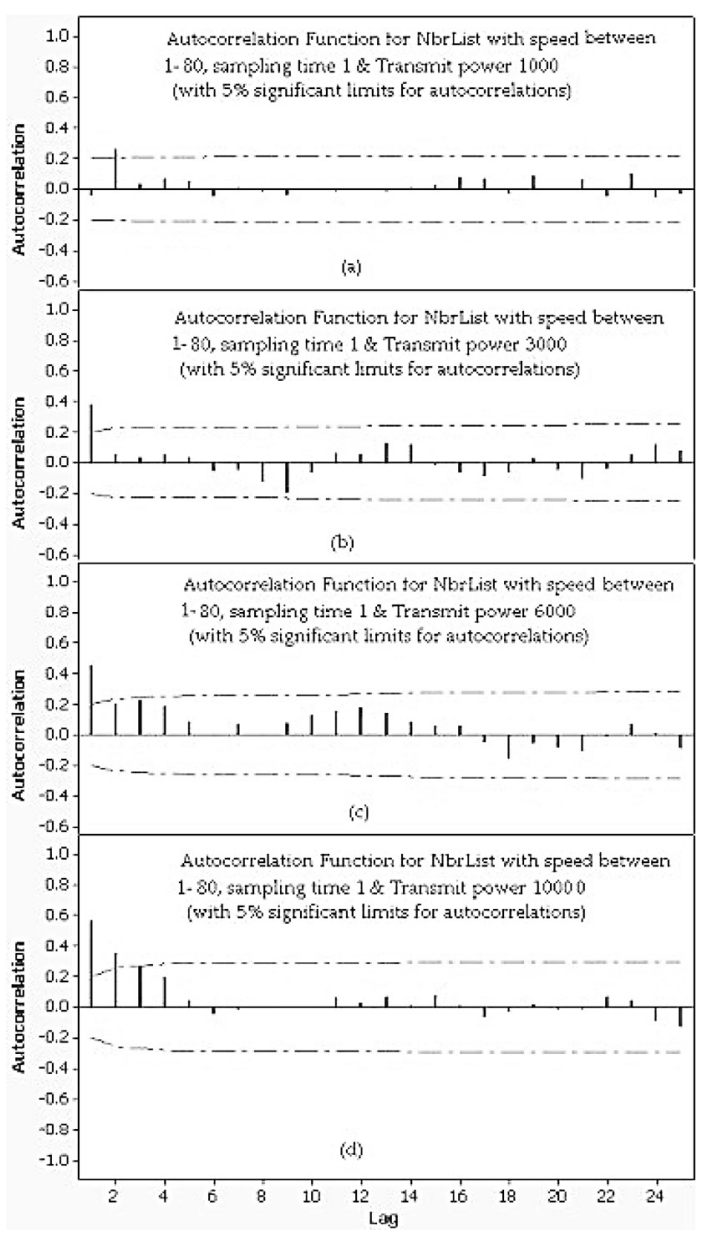

Figure 7. Variation of the NC autocorrelation with the transmission power for the Gaussian mobility pattern. random walk and the random way point mobility pattern respectively. The autocorrelations appear more significant for times less than 4 sec for the random walk mobility model and 6 sec for the random way point mobility models, respectively.

\subsection{Analysis for Varying Speed and Vary- ing Sampling Rate}

The next experiment is conducted to investigate the combined effects of speed and sampling time on the autocorrelation of the $\mathrm{NC}$ values for different mobility models. The speed varies from $10 \mathrm{~m} / \mathrm{sec}$ to $100 \mathrm{~m} / \mathrm{sec}$ in increments of $10 \mathrm{~m} / \mathrm{sec}$ and the sampling time varies from 0.5 $\mathrm{sec}$ to $5 \mathrm{sec}$ in increments of $0.5 \mathrm{sec}$. The observations are summarized in Table 1 . As evident from Table 1, the NC data for nodes moving at the speed of $10 \mathrm{~m} / \mathrm{sec}$ has significant autocor-

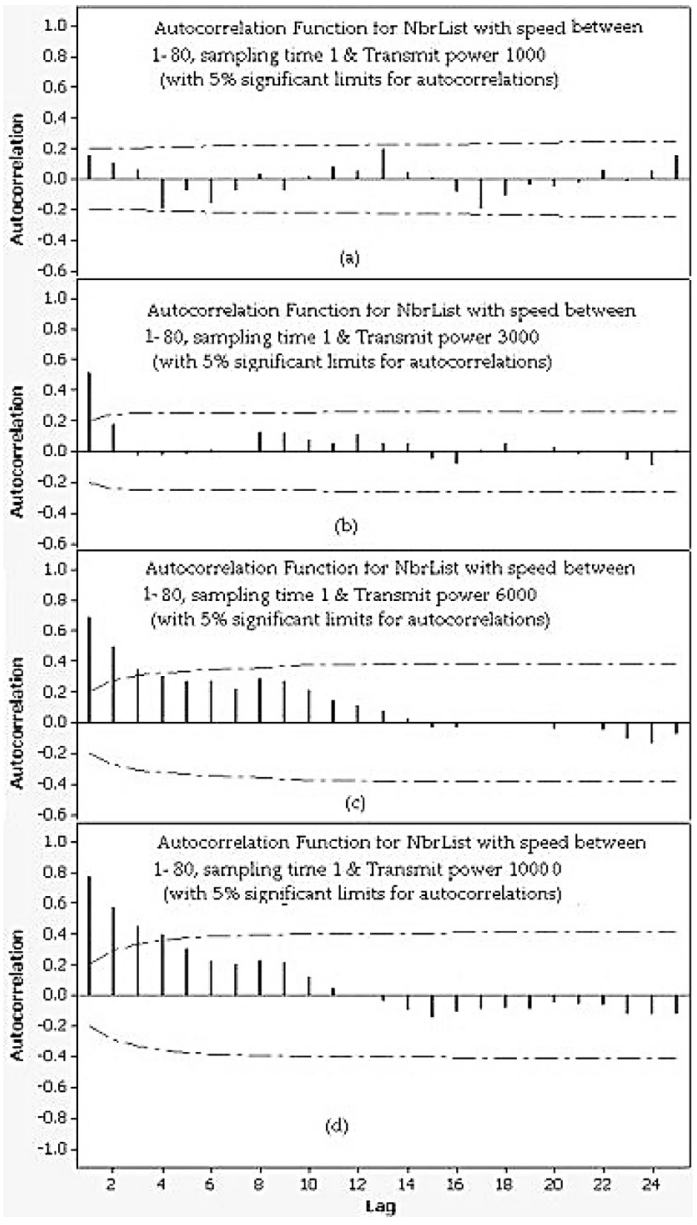

Figure 8. Variation of the NC autocorrelation with the transmission power for the random walk mobility pattern. 


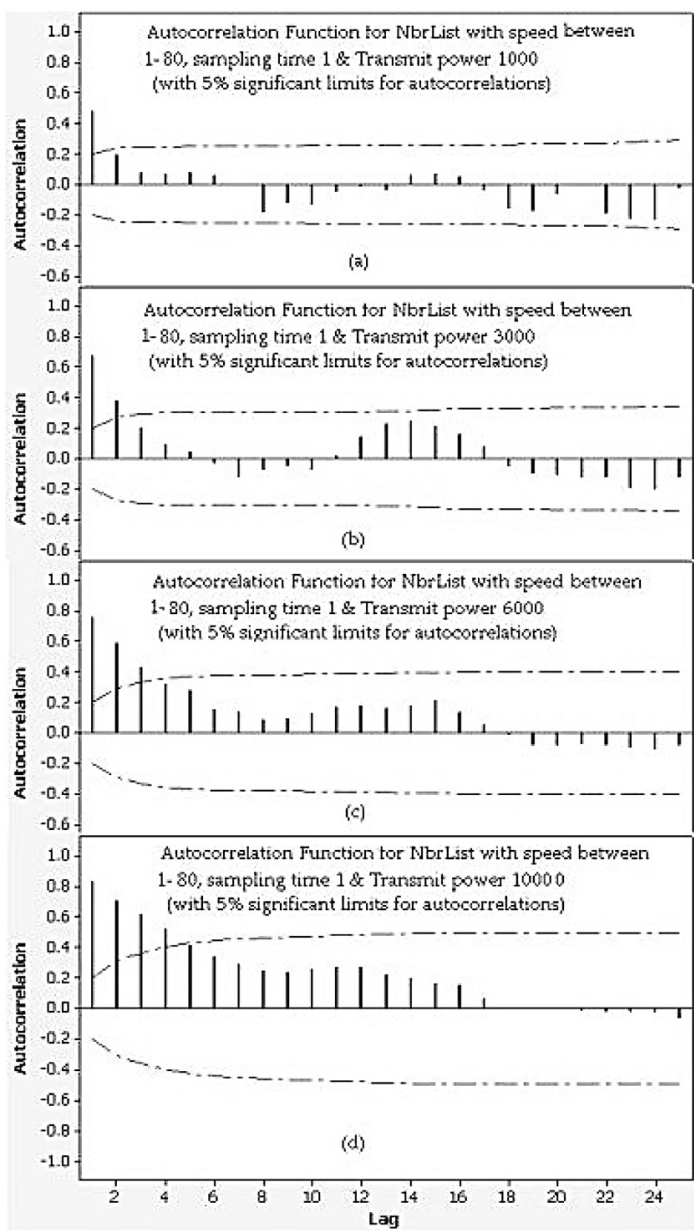

Figure 9. Variation of the NC autocorrelation with the transmission power for the random way point mobility pattern.

relations for each sampling period from 0.5 to $5 \mathrm{sec}$. When the speed reaches $50 \mathrm{~m} / \mathrm{sec}$, the autocorrelations are significant only up to the sampling period of $3.5 \mathrm{sec}$; beyond this sampling time, no significant autocorrelations can be observed. This behavior is expected as the distance covered by the node is a product of the speed and time. Hence, for moderate speeds, if the sampling time is increased, more nodes may move out of the transmission range and similarly more nodes may move in which lower the autocorrelation with the values at previous lags.

\subsection{Analysis for Varying Transmission Range}

The radio range is determined by the transmitting powers of each node. For our experiment, we define the transmission range as the square root of the transmitting power. We have varied the transmitting powers form $1000 \mathrm{~m}$ to 90000 $\mathrm{m}$. For low transmitting powers, no significant autocorrelations are observed between nodes because few nodes are expected in the transmission range. The nodes moving very slowly will also change their neighbors rapidly, effectively removing the correlations. However, as we increase the transmitting powers, the autocorrelations between the nodes start to exhibit sinusoidal patterns with high peaks at the beginning, as shown in Figure 7. From Figure 7.a, we can find that the autocorrelation is not significant for the NC data when the transmission power is $1000 \mathrm{~m}$ for the Gaussian node mobility. The autocorrelations become significant only after the transmission powers are above $3000 \mathrm{~m}$. The autocorrelations for the random walk and the random way point mobility patterns are shown in Figures 8 and 9 respectively. The autocorrelations become also significant for the transmission ranges beyond $3000 \mathrm{~m}$ for the random walk and beyond $2000 \mathrm{~m}$ for the random way point, respectively.

\subsection{Comparison for Different Mobility Patterns}

As we have already pointed out, the threshold for the autocorrelation across different mobility patterns is negligibly small. The threshold values for the autocorrelations across different mobility patterns are tabulated in Table 3 . Table 2 shows the threshold for varying transmission ranges and different mobility patterns. We observe that the transmission power requirements for the Gaussian mobility pattern are higher than for other mobility patterns, however, not by a significant amount. Table 3 contains the threshold data of the autocorrelation for the maximum speed. The random way point mobility model $[17,16,6]$ is very robust for the AR modeling and also has good autocorrelation, even for larger transmission ranges and speeds. As expected, the random way point mobility patterns show good autocorrelation for higher sampling times and higher speeds.

The reason why the random way point mobility model has better autocorrelation properties is that it uses pause times between changes in the node movement directions and speeds, whereas the random walk and the Gaussian mobility models do not use any such pause time. The 
changes in location of nodes within a specific period of time are very high and thus the correlations among the nodes are low. On the other hand, for the random way point mobility model, the changes in location are low compared to other mobility models, so that the correlations among nodes are quite large, as indicated by our results tabulated in Tables 2, 3 and 4.

\begin{tabular}{|c|c|c|c|c|c|c|c|c|c|c|}
\hline speed & $\mathbf{0 . 5}$ & $\mathbf{1}$ & $\mathbf{1 . 5}$ & $\mathbf{2}$ & $\mathbf{2 . 5}$ & $\mathbf{3}$ & $\mathbf{3 . 5}$ & $\mathbf{4}$ & $\mathbf{4 . 5}$ & $\mathbf{5}$ \\
\hline \hline 10 & $\mathrm{y}$ & $\mathrm{y}$ & $\mathrm{y}$ & $\mathrm{y}$ & $\mathrm{y}$ & $\mathrm{y}$ & $\mathrm{y}$ & $\mathrm{y}$ & $\mathrm{y}$ & $\mathrm{y}$ \\
\hline 20 & $\mathrm{y}$ & $\mathrm{y}$ & $\mathrm{y}$ & $\mathrm{y}$ & $\mathrm{y}$ & $\mathrm{y}$ & $\mathrm{y}$ & $\mathrm{y}$ & $\mathrm{y}$ & $\mathrm{y}$ \\
\hline 30 & $\mathrm{y}$ & $\mathrm{y}$ & $\mathrm{y}$ & $\mathrm{y}$ & $\mathrm{y}$ & $\mathrm{y}$ & $\mathrm{y}$ & $\mathrm{y}$ & $\mathrm{y}$ & $\mathrm{y}$ \\
\hline 40 & $\mathrm{y}$ & $\mathrm{y}$ & $\mathrm{y}$ & $\mathrm{y}$ & $\mathrm{y}$ & $\mathrm{y}$ & $\mathrm{y}$ & $\mathrm{y}$ & $\mathrm{y}$ & $\mathrm{y}$ \\
\hline 50 & $\mathrm{y}$ & $\mathrm{y}$ & $\mathrm{y}$ & $\mathrm{y}$ & $\mathrm{y}$ & $\mathrm{n}$ & $\mathrm{n}$ & $\mathrm{n}$ & $\mathrm{n}$ & $\mathrm{n}$ \\
\hline 60 & $\mathrm{y}$ & $\mathrm{y}$ & $\mathrm{y}$ & $\mathrm{n}$ & $\mathrm{n}$ & $\mathrm{n}$ & $\mathrm{n}$ & $\mathrm{n}$ & $\mathrm{n}$ & $\mathrm{n}$ \\
\hline 70 & $\mathrm{y}$ & $\mathrm{y}$ & $\mathrm{y}$ & $\mathrm{n}$ & $\mathrm{n}$ & $\mathrm{n}$ & $\mathrm{n}$ & $\mathrm{n}$ & $\mathrm{n}$ & $\mathrm{n}$ \\
\hline 80 & $\mathrm{y}$ & $\mathrm{y}$ & $\mathrm{n}$ & $\mathrm{n}$ & $\mathrm{n}$ & $\mathrm{n}$ & $\mathrm{n}$ & $\mathrm{n}$ & $\mathrm{n}$ & $\mathrm{n}$ \\
\hline 90 & $\mathrm{y}$ & $\mathrm{y}$ & $\mathrm{n}$ & $\mathrm{n}$ & $\mathrm{n}$ & $\mathrm{n}$ & $\mathrm{n}$ & $\mathrm{n}$ & $\mathrm{n}$ & $\mathrm{n}$ \\
\hline 100 & $\mathrm{y}$ & $\mathrm{y}$ & $\mathrm{n}$ & $\mathrm{n}$ & $\mathrm{n}$ & $\mathrm{n}$ & $\mathrm{n}$ & $\mathrm{n}$ & $\mathrm{n}$ & $\mathrm{n}$ \\
\hline 110 & $\mathrm{y}$ & $\mathrm{n}$ & $\mathrm{n}$ & $\mathrm{n}$ & $\mathrm{n}$ & $\mathrm{n}$ & $\mathrm{n}$ & $\mathrm{n}$ & $\mathrm{n}$ & $\mathrm{n}$ \\
\hline 120 & $\mathrm{n}$ & $\mathrm{n}$ & $\mathrm{n}$ & $\mathrm{n}$ & $\mathrm{n}$ & $\mathrm{n}$ & $\mathrm{n}$ & $\mathrm{n}$ & $\mathrm{n}$ & $\mathrm{n}$ \\
\hline
\end{tabular}

Table 1. Changes in the NC autocorrelation with time and with speed for the Gaussian mobility pattern.

\begin{tabular}{|c|c|c|}
\hline Mobility Pattern & Power & Range \\
\hline \hline Gaussian & 3000 & $54.77 \mathrm{mts}$. \\
\hline Random Walk & 3000 & $54.45 \mathrm{mts}$. \\
\hline Random WayPoint & 2000 & $44.72 \mathrm{mts}$. \\
\hline
\end{tabular}

Table 2. Comparison of the thresholds for varying transmission ranges and powers and different mobility patterns.

\begin{tabular}{|c|c|}
\hline Mobility Pattern & Speed (m/sec) \\
\hline \hline Gaussian & 120 \\
\hline Random Walk & 130 \\
\hline Random WayPoint & 200 \\
\hline
\end{tabular}

Table 3. Comparison of the thresholds for different speeds and different mobility patterns.

\begin{tabular}{|c|c|}
\hline Mobility Pattern & Sampling Time \\
\hline \hline Gaussian & 3.5 \\
\hline Random Walk & 4 \\
\hline Random Way Point & 6 \\
\hline
\end{tabular}

Table 4. Comparison of the thresholds for different sampling times and different mobility patterns.

\begin{tabular}{|c|c|}
\hline AIC value & Order of AR model \\
\hline \hline 3.86160 & 1 \\
\hline 3.84039 & 2 \\
\hline 3.84017 & 3 \\
\hline 3.84902 & 4 \\
\hline
\end{tabular}

Table 5. Comparison of the thresholds for varying sampling times and for different mobility patterns.

\section{The Order of the AR model}

As evident from our numerical experiments, for values of the nodes speed, sampling period, and transmission power being above a given threshold, the NC data show good autocorrelation with the high peaks at initial lags which decrease for higher lags. This behavior confirms that for the given threshold values, the modeling of the $\mathrm{NC}$ data using an autoregressive model is well justified. However, autoregressive modeling requires to determine the order $p$ of the model. Therefore, we consider how to obtain the AR model order $p$ next. We recall that the $\operatorname{AR}(p)$ model is then used to predict the future values of the neighbor counts.

In order to calculate the order of the AR process, we have to obtain the PACF which indicates the most likely value of $p$. We have also used the AIC for confirmation. The PACF of the NC values for nodes moving at speeds $50 \mathrm{~m} / \mathrm{sec}$ is shown in Figure 10 and the corresponding AIC values are given in Table 5. Correspondingly, we have that the $\mathrm{NC}$ data can be modeled using the model $\operatorname{AR}(2)$. On the other hand, using the AIC values, we obtain the order 3 . We have chosen the value of $p$ to be 2 .

We have used the $\operatorname{AR}(2)$ model to predict the future values of NC. The comparison of the predicted and the original data obtained from our experiment are shown in Figure 11. It is evident from Figure 11 that the actual data and the predicted data values are very close. Hence, we formulate the following hypothesis:

$\mathrm{H}_{0}$ : The expected values and predicted values match

$\mathrm{H}_{1}$ : The expected values and predicted values do not match

To test which of the hypothesis is satisfied, we have used the $\chi^{2}$ test. The test criterion for 
$\chi^{2}$ test is $\chi_{0}^{2}=\sum_{i=1}^{n} \frac{\left(O_{i}-E_{i}\right)^{2}}{E_{i}}$ where $O_{i}$ refers to the observed values and $E_{i}$ refers to the expected values. For the data shown in Figure 11, the computed $\chi_{0}^{2}$ value is 6.14 which is much smaller than the value of $\chi_{0.05,9}^{2}$ with 9 degree of freedom at $5 \%$ level of significance. Thus, the hypothesis that the predicted value is similar to the actual value may be accepted with $95 \%$ confidence.

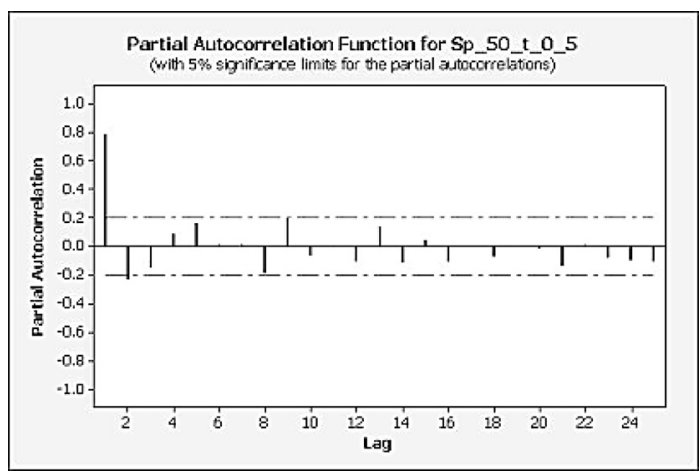

Figure 10. The PACF of the sample NC data values for the Gaussian mobility pattern.

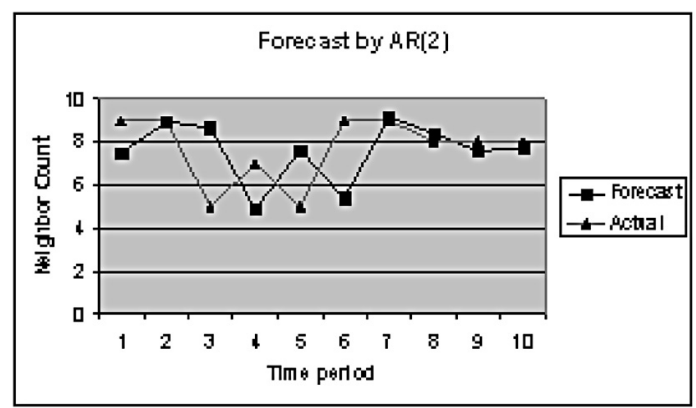

Figure 11. The predicted value of the NC using the $\mathrm{AR}(2)$ model for the Gaussian mobility pattern.

\section{Conclusion}

In this article, we have modeled the temporal neighbor distribution of nodes in mobile ad-hoc network using an autoregressive $\operatorname{AR}(p)$ model often used in stationary time series analysis. We found through our experiments that the node distribution for threshold values of speed, transmission range and sampling time for all three mobility models considered have large correlations of the NC values. The NC correlations were found to be well represented by the $\operatorname{AR}(p)$ model for a suitable choice of $p$. However, above the threshold value, the autocorrelations of the node distribution are not very significant. The thresholds of different parameters were determined and tabulated. The threshold values obtained are suitable for any practical deployment of ad-hoc networks. We have also predicted the number of neighboring nodes in future times and found that these predictions are close to the measured values. The predicted values of neighboring nodes can be used for different network services such as multi-path routing, congestion control, topology construction and traffic prediction. The future work will consider how to incorporate the predicated values of $\mathrm{NC}$ to improve the properties and performances of routing protocols.

\section{References}

[1] N. D. Alparslan, S. KhOSROW, A generalized random mobility model for wireless ad hoc networks and its analysis: One dimensional case. IEEE/ACM Transactions on Networking (TON) 15, 3, 602-615, June 2007.

[2] A. BAnerJeE, P. DutTA, A birth-and-death based mathematical model for tracking mobile nodes in a multihop environment. In BIG, June 2004.

[3] A. Banerjee, K. Majumder, P. Dutta, K. MonDAL, Implementation of the behavior and structure of the multihop mobile environment as a pushdown automata and birth-and-death based statistical model. In Mobile Computing and Networking (MOBICOMNET), pp. 45-51, 2004.

[4] S. Basu, A. Mukherjee, S. Klivansky, Time series models for internet traffic. In INFOCOM, vol. 2, pp. 611-620, March 1996.

[5] C. Bettstetter, Topology properties of ad hoc networks with random waypoint mobility. ACM SIGMOBILE Mobile Computing and Communication Review 7, 3, 50-52, July 2003.

[6] C. Bettstetter, C. Wagner, T. U. München, The node distribution of the random waypoint mobility model for wireless ad hoc networks. IEEE Transactions on Mobile Computing 2, 257-269, 2003.

[7] Y. Borgne, S. SAntini, G. Bontempi, Adaptive model selection for time series prediction in wireless sensor networks. International Journal for Signal Processing 87, 12, 3010-3020, 2007.

[8] P. J. BrockWEll, R. DAVIS, Time Series: Theory and Methods. Springer-Verlag, New York, 1987. 
[9] T. CAMP, J. Boleng, V. Davies, A survey of mobility models for ad hoc network research. Wireless Communications and Mobile Computing (WCMC): Special issue on Mobile Ad Hoc Networking: Research, Trends and Applications 2, 5, 483-502, 2002.

[10] M. Gerla, J. TSAI, Multicluster, mobile, multimedia radio network. IEEE Journal of Wireless Networks 1, 3, 255-265, 1995.

[11] S. Gowrishankar, T. Basavraju, S. K. SARKAR, Effect of random mobility models pattern in mobile ad hoc networks. International Journal of Computer Science and Network Security 7, 6, June 2007.

[12] J. Hamilton, Time Series Analysis. Princeton University Press, Princeton, New Jersy, 1994.

[13] W. Heinzelman, A. Chandrakasan, H. BAlaKRISHNAN, An application-specific protocol architecture for wireless microsensor networks. IEEE Transactions on Wireless Communications 1, 4, 660-670, October 2002.

[14] R. HEKMAT, Ad-hoc Networks:Fundamental Properties and Network Topologies. Springer, AA Dordrecht, The Netherlands, 2006.

[15] D. Herbert, G. Modelo-Howard, C. PerezTORO, S. BAGCHI, Fault tolerant arima-based aggregation of data in sensor networks. In IEEE International Conference on Dependable Systems and Networks, June 2007.

[16] E. Hyytia, P. LAssila J. ViRTAmo, A markovian waypoint mobility model with application to hotspot modeling. In IEEE International Conference on Communications, vol. 3, pp. 979-986, 2006.

[17] P. Lassila, E. Hyytï̈, H. Koskinen, Connectivity properties of random waypoint mobility model for ad hoc networks. In MedHoc-Net, Uüle de Porquerolles, pp. 159-168, 2005.

[18] H. LI, D. YU, A statistical study of neighbor node properties in ad hoc network. In ICPPW'02: Proceedings of the 2002 International Conference on Parallel Processing Workshops (Washington, DC, USA, 2002), IEEE Computer Society, pp. 103-109.

[19] C. LIU, K. Wu, M. TSAO, Energy efficient information collection with the arima model in wireless sensor networks. In IEEE Global Telecommunications Conference GLOBECOM-05, vol. 5, pp. 2470 2474, 2005.

[20] H.-N. NGuYen, Y. SHINODA, A node's number of neighbors in wireless mobile ad hoc network: a statsitical view. In International Conference on Networks ICN 2009, pp. 52-60, 2009.

[21] T. Soong, Fundamentals of probablilty and statistics for engineers. John Wiely and Sons Ltd., Sussex, England, 2004.

[22] A. VARGA, The omnet ++ discrete event simulation system. In The European Simulation Multiconference (ESM2001), pp. 319-324, 2001.
[23] J. Yoon, M. LIU, B. Noble, Random waypoint considered harmful. In INFOCOM 2003, pp. 13121321, 2003.

[24] C. You, K. Chandra, Time series models for internet data traffic. In Local Computer Networks (LCN), pp. 164-171, 1999.

[25] Q. ZHENG, X. Hong, S. RAY, Recent advances in mobility modeling for mobile ad hoc network research. In ACM Southeast Regional Conference, ACM New York, NY, USA, pp. 70-75, 2004.

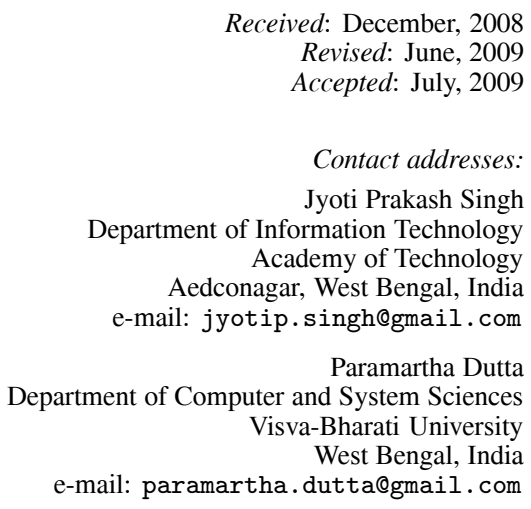

JYOTI PRAKASH SINGH did his B.Tech in Computer Science and Technology from Kalyani Government Engineering College, West Bengal, India in the year 2000. He completed his M.Tech in Information Technology in the year 2005 from Sikkim Manipal Institute of Technology, Sikkim, India. He is currently serving as an Assistant Professor in the Department of Information Technology in Academy of Technology Bandel, West Bengal, India. He has about 15 publications in the area of ad hoc network and data mining in national and international conferences. He is coauthor of the book "Schaum's Outline of Operating System", special Indian edition by Tata McGraw Hill Education, 2008. His current research interests includes sensor ad hoc network and data mining.

PARAMARTHA DUTTA did his Bachelors and Masters in Statistics from Indian Statistical Institute, Kolkata, India in 1988 and 1990 respectively. Subsequently, he did his Master of Technology in Computer Science in 1993 from Indian Statistical Institute, Kolkata, India. He completed his Ph. D in Engineering in 2005 from Bengal Engineering and Science University, Shibpore, India. He is currently a Professor in the Department of Computer and System Sciences, Visva-Bharati University, Santiniketan, West Bengal, India. He has co-authored 4 books and has 1 edited book to his credit. Apart from this, he has more than 65 research publications in various national and international journals and conference proceedings. His research interests include evolutionary computation, soft and intelligent computing, pattern recognition and mobile computing. Dr. Dutta is a Fellow of Optical Society of India. He is a Life Member of Indian Science Congress Association, Computer Society of India, Institution of Electronics and Telecommunication Engineering, Indian affiliate of International Association of Pattern Recognition, Indian Society for Technical Education and International Association of Engineers, Hong Kong. He is also a Member of Association for Computing Machinery, USA. 
